\title{
Evaluation of the pharmacoeconomics of drugs used for the treatment of long-term complications of sulfur mustard
}

\author{
Yunes Panahi, ${ }^{1}$ Mostafa Ghanei, ${ }^{1}$ Milad Vakili Zarch, ${ }^{2}$ Zohreh Poursaleh, ${ }^{1}$ Shahram Parvin, ${ }^{1}$ Ramin Rezaee, ${ }^{3}$ \\ Amirhossein Sahebkar ${ }^{4}$ \\ ${ }^{1}$ Chemical Injuries Research Center, Baqiyatallah University of Medical Sciences, Tehran; ${ }^{2}$ School of Medicine, Isfahan \\ University of Medical Sciences, Isfahan; ${ }^{3}$ Department of Physiology and Pharmacology, School of Medicine, North Khorasan \\ University of Medical Sciences, Bojnurd; ${ }^{4}$ Biotechnology Research Center, Mashhad University of Medical Sciences, Mashhad, \\ Iran
}

\begin{abstract}
Sulfur Mustard (SM), a cytotoxic vesicant chemical warfare agent, has powerful irritant and blistering effects on the skin, eyes and respiratory tract. Since during the Iraq-Iran war, many Iranian soldiers and civilians were exposed to SM, there are several victims still suffering from long-term cutaneous, ocular and pulmonary complications. Currently, there is no definite treatment for long-term complications of SM, and only supportive medical care is being taken to minimize the symptoms. In this study, we compared the cost-effectiveness of common drugs that are used against long-term SM-induced complications in Iranian patients. In this review article, electronic databases were checked using the following key words: sulfur mustard, lung, skin, eye, cost-effectiveness, pharmacoeconomics and treatment. Abstracts of non-English papers and proceedings of congresses on SM were also assessed. Among the studied drugs, high-dose oral N-acetyl cysteine and long-acting inhaled corticosteroids against respiratory complications, topical corticosteroids and oral antihistamines against cutaneous complications and non-steroidal anti-inflammatory drugs and corticosteroids ophthalmic drops against ocular complications were found to be cost-effective. Usage of different drugs in the treatment of SM injuries in Iran, have imposed a significant economic burden to patients and their families because many drugs that are effective against chemical injuries are not covered by insurance. In addition, the development of more effective drugs in this field is considered as an urgent demand that should be noticed by the pharmaceutical industry.
\end{abstract}

\section{Introduction}

Sulfur Mustard (SM) is an alkylating agent widely used in chemical warfare. For the first time, SM was

Correspondence: Amirhossein Sahebkar, Biotechnology Research Center, Mashhad University of Medical Sciences, Mashhad, Iran.

Tel.: +98.511.8823255 - Fax: +98.511.8823251.

E-mail: sahebkarah811@mums.ac.ir ; sahebkara@mums.ac.ir ; amir_saheb2000@yahoo.com

Key words: Sulfur mustard; lung; skin; eye, cost-effectiveness; treatment; pharmacoeconomics.

Conflict of interest: the authors declare no potential conflict of interest.

Received for publication: 17 May 2016.

Revision received: 1 July 2016.

Accepted for publication: 17 August 2016.

This work is licensed under a Creative Commons Attribution NonCommercial 4.0 License (CC BY-NC 4.0).

CCopyright Y. Panahi et al., 2017

Licensee PAGEPress, Italy

Italian Journal of Medicine 2017; 11:102-113

doi:10.4081/itjm.2016.743 used by the German military forces at Ypresin in September 1917 during World War I. More recently, SM was used in the Iraq-Iran war (1980-1988). The world's first city in which civilians were attacked with chemical weapons was Sardasht which is located in the North West of Iran. ${ }^{1}$

When SM is released into the air, it can be transferred by wind affecting people over a vast area. SM can enter our body by inhalation, absorption from the skin or through the anterior surface of the eyes and also through the gastrointestinal tract from contaminated food or water. The effects depend on the severity and duration of the exposure and usually become apparent about $12 \mathrm{~h}$ after the exposure. SM-induced mortality is usually low and in severe cases, death can occur 4-5 weeks after the exposure. Only very high doses of SM (64 SM/kg dermal exposure or 1500 $\mathrm{SM} / \mathrm{min} \times \mathrm{m}^{3}$ inhalation) are acutely fatal in humans in a time interval as short as one hour. Fatality rates among the exposed soldiers during World War I and casualties of the Iran-Iraq war were about $2 \%$ and 3 $4 \%$, respectively. ${ }^{2}$

During the Iran-Iraq war, many Iranian military personnel and civilians were exposed to SM. There is no definite treatment for long-term complications of SM, and SM-poisoned individuals receive supportive medical care that minimizes the effects of the expo- 
sure. The aim of the current study was to compare a cost-effectiveness of drugs that are being commonly used against long-term complications of SM in Iranian patients.

\section{Methods of research}

All studies published until June 2013 that focused on the treatment of lung, skin and eye injuries following SM exposure, were included. Publications were obtained from the following electronic databases: Medline/Pubmed, Scopus, Google Scholar, Embase, ISI Web of Knowledge, Biological Abstracts and Chemical Abstracts. To include all of the studies indexed in electronic databases, keywords such as sulfur mustard, lung, skin, eye, cost-effectiveness, treatment and pharmacoeconomics were used. Subsequently, therapeutic approaches for SM-induced pulmonary, skin and eye injuries were analyzed. Also, in this study, efficient prophylactic/therapeutic measures against acute and long-term SM-induced pulmonary damages are classified. To evaluate the cost-effectiveness of the drugs, the prices of medications that are being commonly used against SM-induced pulmonary, dermal and ocular complications were also studied. Parameters of clinical efficacy of common drugs were obtained from the literature and local standard costs, adverse events, and micro/macrovascular complications were considered in this regard. Drug prices were provided by the ministry of health and medical education of Islamic Republic of Iran (www.behdasht. gov.ir), Iran health insurance organization, Social security organization of Islamic Republic of Iran (www.tamin.ir) and Social organization of the armed forces of Islamic Republic of Iran. Prices (updated in June 2013) are reported in Rials.

\section{Lung injury}

Respiratory system is an important target of SM and if it is injured by SM, higher morbidity and mortality will be expected. ${ }^{1}$ The greatest discomfort produced by SM is the respiratory system injury.

\section{Acute lung injury}

Few weeks after SM exposure, the common cause of discomfort and fatalities is respiratory system irritation and injury. ${ }^{3}$ Respiratory effects are dose-dependent and can be seen in all parts of the respiratory system from the nasal mucosa to the terminal bronchioles. As SM has a high chemical activity, most of the acute injuries are limited to the upper respiratory tract. ${ }^{4}$

Recovery from the acute injury can be rapid but some irritation, cough, and huskiness may persist for about 6 weeks. However, a 1-2-month delay in recov- ery happens mostly after secondary infections and necrotic bronchopneumonia. ${ }^{3}$

\section{Chronic lung injury}

Respiratory problems are the most common longterm consequences observed among patients with SM exposure. Three years after SM exposure, a triad of cough, expectoration and dyspnea was shown in most of Iranian victims. ${ }^{5}$

Chronic obstructive pulmonary disease is more common than restrictive patterns. ${ }^{5}$ Chronic laryngitis, tracheobronchial stenosis, tracheobronchomalacia, chronic bronchitis, emphysema, bronchiectasis, pulmonary fibrosis, bronchiolitis obliterans and asthma are well-known chronic consequences of SM exposure among Iranian victims. However, alveolar microlithiasis, mediastinal emphysema and unilateral lung collapse may infrequently occur. ${ }^{1,2}$

Unlike other chronic effects of SM, pulmonary dysfunctions worsen over time. ${ }^{2}$ Major causes of SMinduced morbidity and mortality are bronchial pneumonia and septicemia secondary to opportunistic infections of the injured respiratory tract. ${ }^{3,6}$ Also, a single exposure to SM may increase the risk of lung cancer in some individuals. ${ }^{7}$

\section{Management of respiratory toxic effects and cost-effectiveness of drugs used in the treatment of sulfur mustard-induced respiratory complications}

Lung injuries due to SM are associated with protease activation, oxidative injury and inflammatory responses. Previous studies on SM demonstrated the role of oxidative stress in SM toxicity and suggested antioxidant agents as effective treatments to decrease injuries. Chronic bronchitis is the most common chronic respiratory disease in SM-exposed Iranians.

Study of Ghanei et al. on SM-exposed patients who had chronic bronchitis and were not responsive to standard treatments in exacerbation occasions, showed that there was a significant improvement in spirometric indices of patients receiving short-term intravenous or oral corticosteroids. ${ }^{8}$

In another study, results revealed that a 6-month treatment with interferon-gamma- $1 \mathrm{~b}$ in combination with low-dose prednisolone improves lung function tests in SM-exposed patients who had bronchiolitis. ${ }^{9}$ Ghanei et al. suggested that inhaled corticosteroids and long-acting $\beta 2$-agonists are effective in the treatment of SM-exposed patients who had chronic bronchiolitis. Also, a medium dose of fluticasone/salmeterol had the same effects on the airways. ${ }^{10}$

As previous studies revealed, oxygen species and free radicals contribute to pulmonary damages caused 
by SM. So, it will be useful to consider antioxidant drugs in the management of these injuries. $\mathrm{N}$-acetyl cysteine (NAC) is a mucolytic drug with antioxidant activities. Prescription of NAC may be effective in the treatment of diseases caused by reactive oxygen species. Shohrati et al. showed that 4-month administration of NAC (1800 $\mathrm{mg}$ daily) can improve clinical outcomes and spirometric findings in SM-exposed patients. ${ }^{11}$

Ghanei et al. in a study on SM-induced bronchiolitis obliterans with normal pulmonary function test, revealed that a 4-month trial of oral NAC is effective against bronchitis and also bronchiolitis. They reported that NAC prevented SM-induced oxidative stress, and can be used in the treatment of pulmonary diseases in SM-exposed patients. ${ }^{12}$

Another study confirmed previous results and revealed that the instillation of liposomes containing reducing agents like NAC can significantly reduce acute lung injury even when instillation was delayed as long as 1 hour after the exposure of lungs to SM. ${ }^{13}$ Dyspnea is one of the most important complaints of SM-exposed patients with chronic obstructive pulmonary disease. Shohrati et al. showed that nebulized morphine can improve dyspnea, coughing, respiratory rate, heart rate, night-time awaking secondary to dyspnea, night-time awaking due to coughing and peak expiratory flow rate without any important side effects. ${ }^{14}$

Also, it was shown that inhalation of furosemide is not different from placebo in improving dyspnea due to pulmonary diseases in SM-exposed patients. So, it was suggested that SM-exposed patients who had chronic bronchitis or bronchiolitis, may not benefit from furosemide to improve dyspnea. ${ }^{15}$ Increased airway responsiveness to $\beta$-agonists is noted in asthmatics and smokers. Also, increased airway responsiveness to salbutamol in most subjects exposed to chemical warfare agents, was shown. ${ }^{16}$

In Table 1 we compared costs of the drugs that are being commonly used against respiratory complications of SM in Iran. Also, we compared the efficacy of drugs based on previous studies. ${ }^{16}$

As shown in Table 1, previous studies on the efficacy of NAC against respiratory complications of SM reported promising results as NAC could reduce the respiratory symptoms in SM-exposed patients. NAC is covered by health insurance of the armed forces of Iran and is of low price for veterans. For this reason, NAC has a high cost-effectiveness in Iranian SM-injured subjects. Inhaled corticosteroids are another group of drugs that have reasonable effects in this respect. $\beta$-agonists, despite the lower price, do not have significant effects on respiratory symptoms in SM-exposed subjects and many patients are resistant to the therapeutic effects of this class of drugs.

Some of the mentioned drugs including interferongamma have high prices and are not affordable for pa- tients despite excellent efficacy. Studies on the efficacy of macrolides (azithromycin, clarithromycin and erythromycin) showed that this group of drugs has good effects on inflammatory complications of SM and reduces symptoms but they (specially clarithromycin) have higher prices as compared to more common drugs and the insurance coverage does not compensate this difference. Prednisolone and similar drugs, despite their low cost and high effectiveness, have many side effects. Therefore, among the listed drugs, NAC appears to have the highest cost-effectiveness.

\section{Skin injury}

\section{Acute skin injury}

Several hours after SM exposure, dermal symptoms become visible. The intensity of lesions depends on the SM concentration. The characteristics of SM skin lesions are erythema and blisters (small vesicles). Rubbing these blisters can produce new blisters. Erythema and sometimes itching occur 2-8 h after exposure. Blisters appear 4-18 h after exposure. Gradually, blisters join together to form the characteristic pendulous blisters containing large volumes of a clear yellow fluid. Usually, blisters are not painful but they may be uncomfortable, feel tense and cause difficult movement and pain if they appear over the joints. ${ }^{17,18}$

These yellow liquid-filled blisters will merge together to form larger bullae. Rupture of the large blisters can cause full-thickness skin loss and ulceration followed by formation of a necrotic layer or scar on the skin surface about $72 \mathrm{~h}$ after the exposure. ${ }^{19,20}$ Two weeks after milder SM exposure, the erythematous areas of skin become hyper-pigmented without other skin lesions. Also, hypo-pigmentation can occur during healing. The burns caused by high-dose exposures are very painful and the full-thickness of skin can be lost. ${ }^{17,21}$

SM-induced burns heal more slowly than a thermal burn wound. SM burns healing latency depends on the burnt surface area. In mild burnings, lesion may be only an erythema, which turns black in about 1015 days. ${ }^{22}$

In Iranian SM victims, skin lesions were categorized as erythematous form, pigmentary exfoliation, superficial vesicular to bullous form, bullous necrotization, deep necrotizing non-bullous form, and allergic and toxic contact reactions of the skin. The most usual acute skin lesions in the Iraq-Iran war victims were erosions, erythema and hyperpigmentation. ${ }^{23,24}$

\section{Chronic skin injury}

Chronic skin injury after SM exposure usually happens in those with a background of blister formation and skin necrosis. The injuries can be disfiguring and impair the quality of patient's life. ${ }^{25}$ 


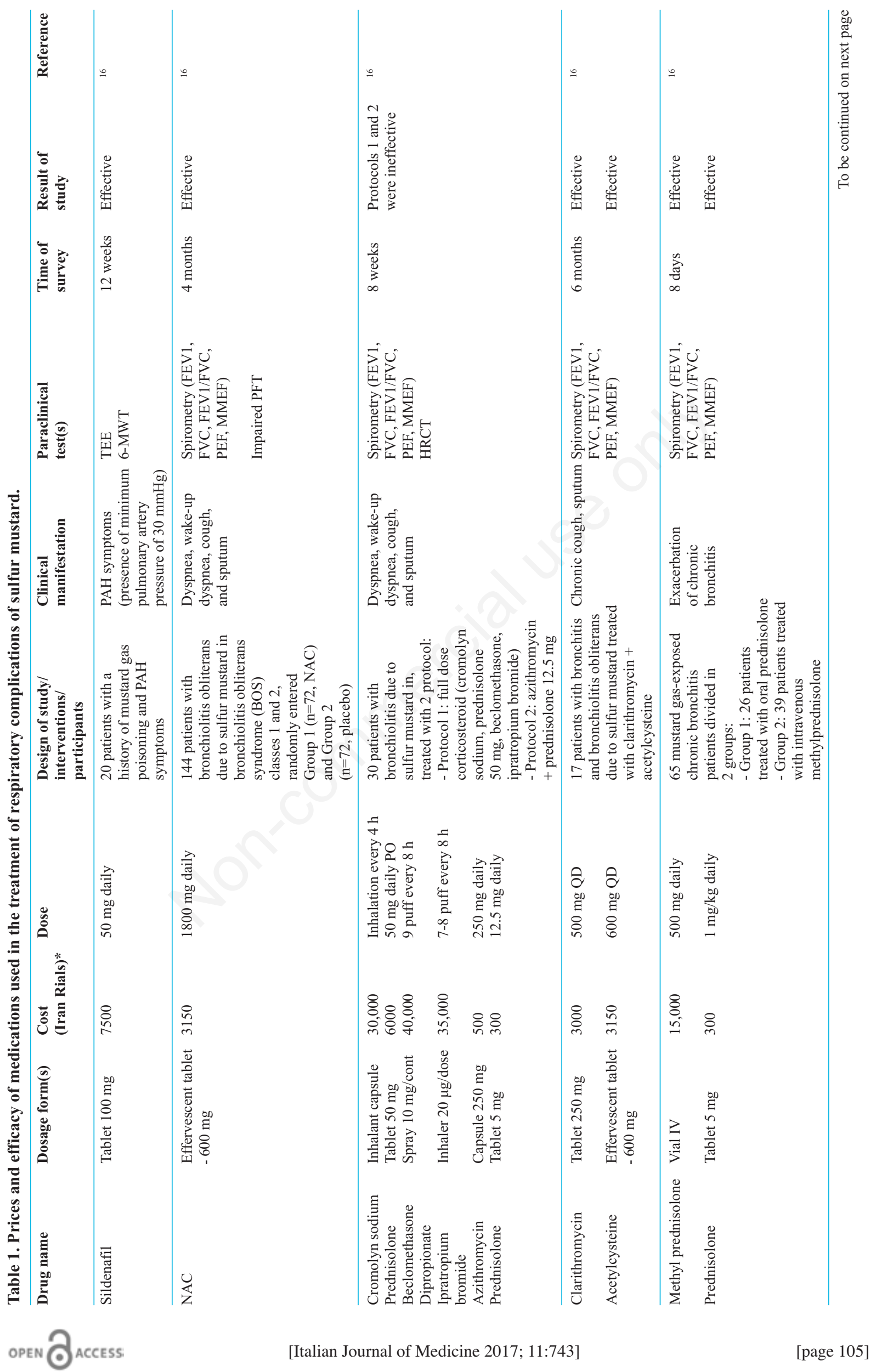




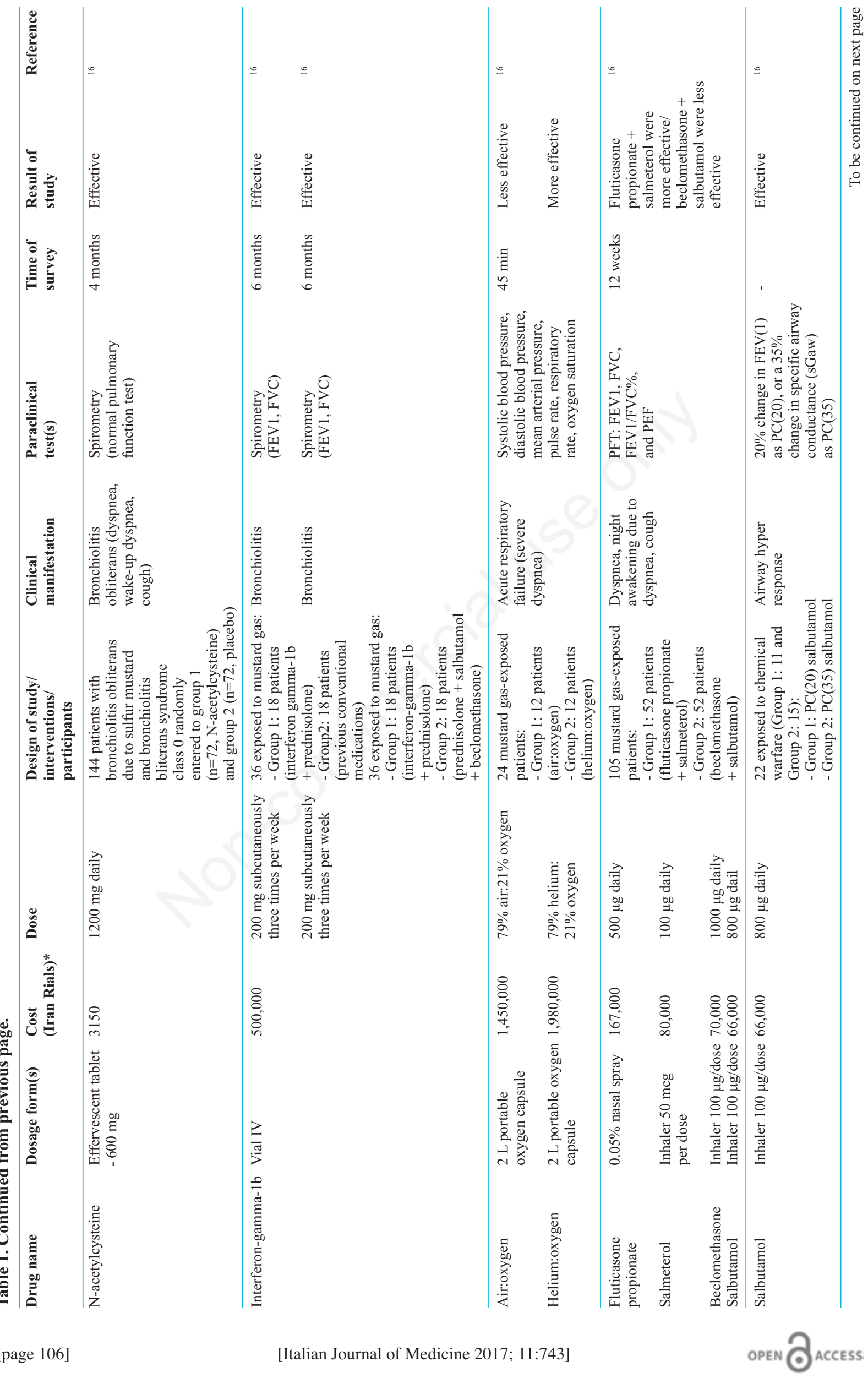




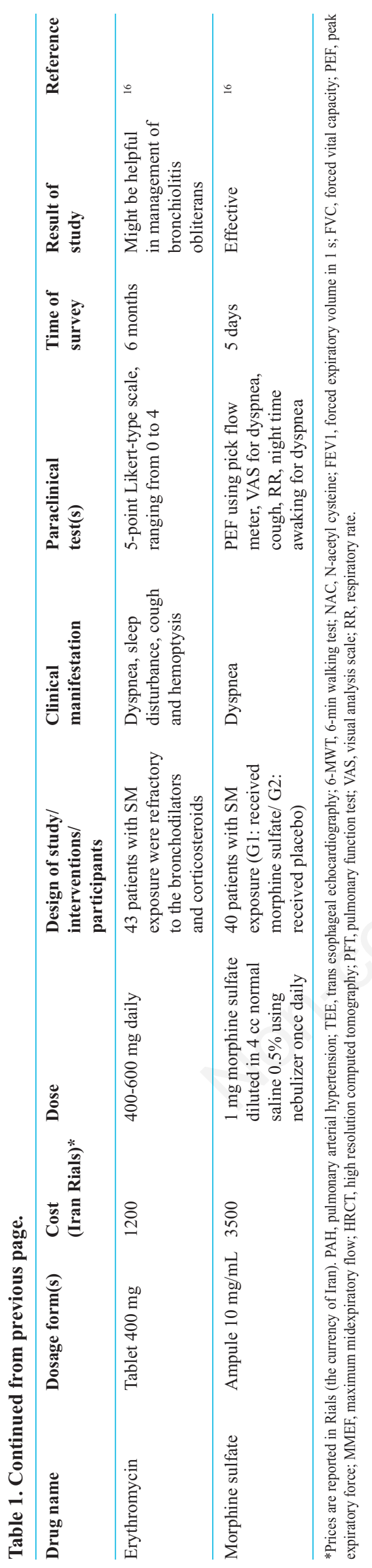

Studies revealed that hyperpigmentation, hypopigmentation and dermal scar are the three most common late skin consequences of SM exposure. Also, local hair loss, eczema and chronic urticaria could be seen. Common skin complaints of these patients were itching, burning sensation and desquamation. These are because of dryness of the skin as they become worse in dry weather and after physical activity. Several years after exposure, pruritus was still the most common symptom. ${ }^{2,19,26}$

The most common skin conditions $16-20$ years post-exposure to SM were hyperpigmentation, erythematous papular rash, dry skin, multiple cherry angiomas, atrophy, hypopigmentation, and hypertrophy. ${ }^{19}$

A higher incidence of vitiligo, psoriasis and discoid lupus erythematous was reported among SM-exposed patients. ${ }^{27} \mathrm{SM}$ has an adverse effect on immune system and these diseases have an immunological basis. ${ }^{28}$ Also, it has been reported that injured sites are sensitive to mechanical stimuli. ${ }^{2}$ The incidence of skin cancers after SM exposure is low and it is not clear whether skin malignancies are associated with SM carcinogenicity. ${ }^{21}$

\section{Management of skin complications of sulfur mustard and cost-effectiveness of drugs}

Erythema, burning, and itching develop after dermal exposure to SM. Cooling these sites relieves the symptoms. Also, hypothermia may lessen the severity of SM-induced cutaneous lesions. ${ }^{29}$

Pain can be treated with analgesics, like acetaminophen and opioids. Anti-histamines and local corticosteroids are useful to reduce itching. ${ }^{17}$ Small vesicles should not be opened; but, it is better to remove the top of larger blisters and apply a sterile dressing on them because of their susceptibility to rupture. ${ }^{30}$

In SM-exposed patients, chronic skin lesions are common and pruritus is the most common complaint, which affects the patients' quality of life. Panahi et al. showed that a combination of phenol and menthol has a significant therapeutic effect on pruritus in SM-exposed victims. ${ }^{31}$

Another study by Panahi et al. revealed that curcumin (a bioactive polyphenol from turmeric ${ }^{32-49}$ ) might be considered as a natural, safe and inexpensive treatment for SM-induced chronic pruritus. Also, they showed that curcumin could improve the quality of life of the victims. ${ }^{36,50-52}$

In another study, Shohrati et al. compared the effect of Unna's Boot cream and betamethasone on pruritus severity in SM-exposed subjects who had chronic cutaneous complications. They showed that there is no significant difference in the improvement of pruritus between the two drugs. Since long-term and widespread use of corticosteroids has restrictions and 
because of limited adverse effects of Unna's Boot cream, Unna's boot seems to be a more reasonable agent for long-term treatment of SM-related pruritus as compared to betamethasone..$^{53}$

Shohrati et al. also showed that hydroxyzine and doxepin had equal results on SM-induced pruritus but they had better effects in comparison with cetirizine in controlling the symptoms of chronic pruritus..$^{54,55}$

Moreover, topical pimecrolimus and betamethasone were shown to be effective in controlling pruritus, burning sensation and also skin dryness but the severity of vesicle, erythema, fissure, lichenification, excoriation, hyperpigmentation and hypopigmentation did not decrease significantly following the administration of the afore-mentioned drugs. Also, they did not report any serious side effects during their study. ${ }^{56}$ Panahi et al. reported equal efficacy for doxepin and betamethasone, and suggested that doxepin can be a potential alternative to betamethasone in controlling SM-induced pruritus. ${ }^{57}$

In a study conducted by Panahi et al., results revealed that Aloe vera/olive oil combination cream was as effective as betamethasone in the treatment of SMinduced chronic skin complications. ${ }^{58}$

In another study, the efficacy of immunotherapy using interferon-gamma (IFN- $\gamma$ ) in the treatment of SM-Induced chronic cutaneous complications was compared with topical betamethasone and the results showed that treatment with IFN- $\gamma$ was associated with greater reductions in atopic dermatitis scores and improvements in life quality, as compared to betamethasone. The authors proposed that application of IFN- $\gamma$ can be effective against SM-induced chronic skin complications. ${ }^{59}$

Additionally, the effects of capsaicin and betamethasone were compared and results revealed that both drugs can significantly decrease pruritus and skin dryness in SM-exposed patients but burning sensation was not improved in capsaicin-treated group. The results also showed that capsaicin topical cream was much less tolerated than betamethasone and also its effect on reducing chronic skin lesions was lesser than that of betamethasone. ${ }^{60}$

In a study done by Panahi et al., some of the patients who received capsaicin, reported a burning sensation and intolerable odor, but these effects were not serious enough to stop the treatment. Capsaicin topical cream $0.025 \%$ was much less tolerated than betamethasone and inferior to betamethasone in reducing SM-induced chronic skin lesions and related symptoms. ${ }^{60}$

Panahi et al. in another study on chronic pruritus in SM-exposed patients reported that curcumin may be regarded as a natural, safe, widely available and inexpensive treatment for the management of SM-induced chronic pruritus. ${ }^{60}$

As shown in Table $2,{ }^{60}$ antihistamines such as hy- droxyzine and doxepin have lower prices and higher efficacies than Unna's Boot and capsaicin cream. Topical corticosteroids also have lower prices than Unna's Boot and capsaicin cream but it should be noted that the efficacies of these drugs are lower.

\section{Eye injury}

\section{Acute eye injury}

The most responsive organs to SM are eyes. It happens because of the high cellular turnover and intense metabolic activity of the corneal epithelial cells and also enhanced penetration through the aqueous-mucous surface of the cornea and conjunctiva. The latent period of initial signs and symptoms are shorter than those seen for skin effects and occur within minutes after attack. ${ }^{61,62}$ Acute intoxication causes conjunctivitis, grittiness under the eyelid, tearing, local edema, blepharospasm, lacrimation, miosis, photophobia and severe eye pain. Conjunctivitis due to mild exposure to SM heals within a few days. At higher doses, fullthickness corneal injury, long-lasting chronic inflammation, delayed-onset lesions, chemical anterior uveitis, posterior synechiae, cataract and permanent blindness are probable (Table 3). ${ }^{18,19,30,63,64}$

\section{Chronic eye injury}

Complaints during chronic phase include itching, burning sensation, photophobia, tearing, decreased vision, dry eye, red eye, ophthalmic pain and foreign body sensation. ${ }^{19,63,65}$ An important ocular phenomenon known as delayed-type ulcerative keratitis may happen in some cases. It is characterized by corneal thinning, opacification, neovascularization and epithelial deficiency leading to photophobia, lacrimation, failing vision and late-onset blindness. Javadi et al. reported that delayed-type ulcerative keratitis usually develop in patients with multiple systemic injuries. ${ }^{19,61,63}$ The pathogenesis of this chronic phenomenon is not clear, but formation of free radicals, by-products toxicity, necrotic changes, damage of the limbal vasculature and autoimmune reactions have been mentioned as possible causes and mechanisms. ${ }^{62,63,66-68}$

\section{Management of eye complications of sulfur mustard and cost-effectiveness of drugs}

About $48 \mathrm{~h}$ after exposure to SM, spontaneous recovery occurs gradually even without treatment and the corneal epithelium is fully healed within a week. If the edema subsides by 1-2 weeks, usually recurrence does not occur. ${ }^{19,64}$ The eyes should be washed immediately after exposure even for asymptomatic pa- 


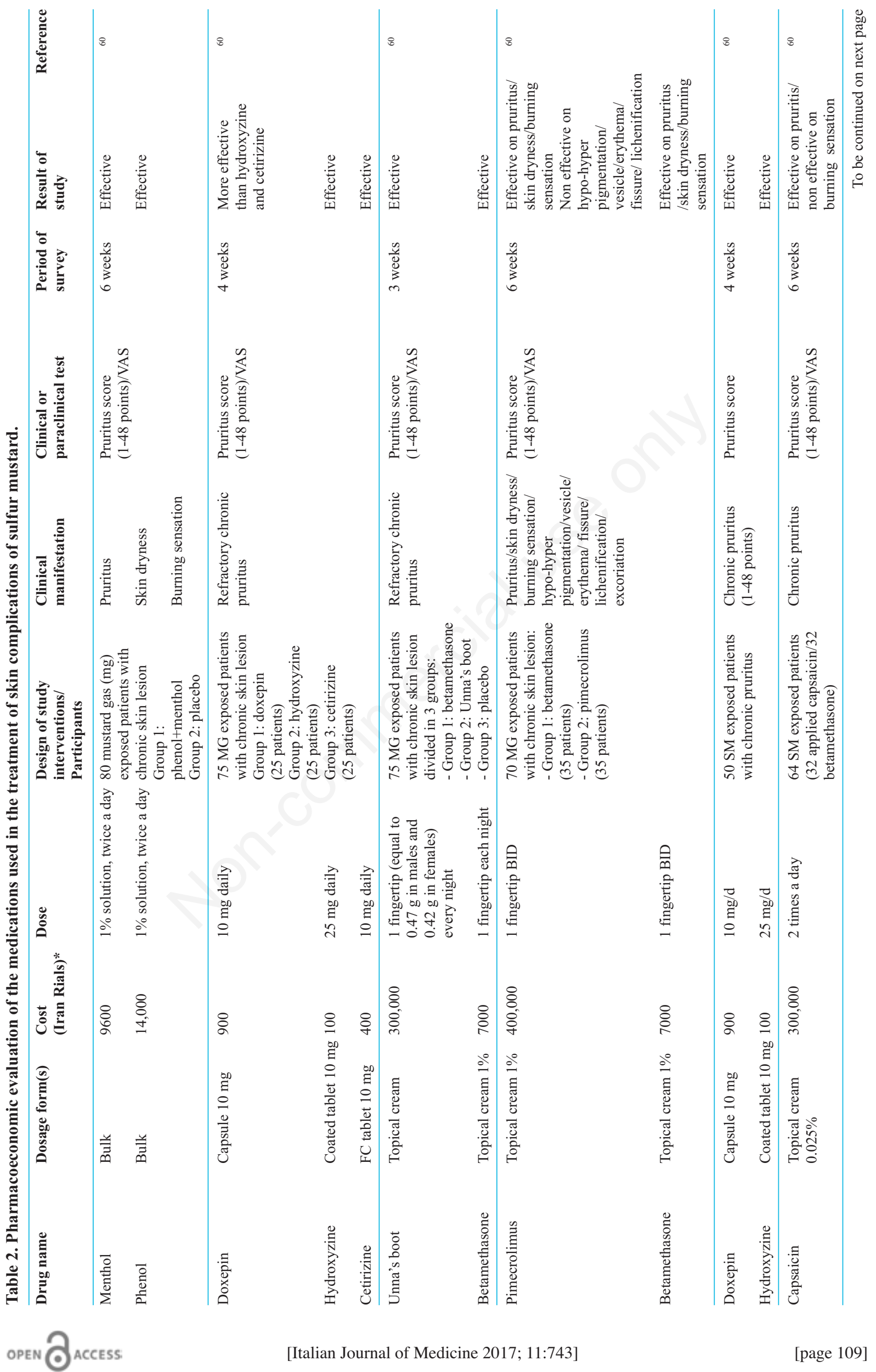




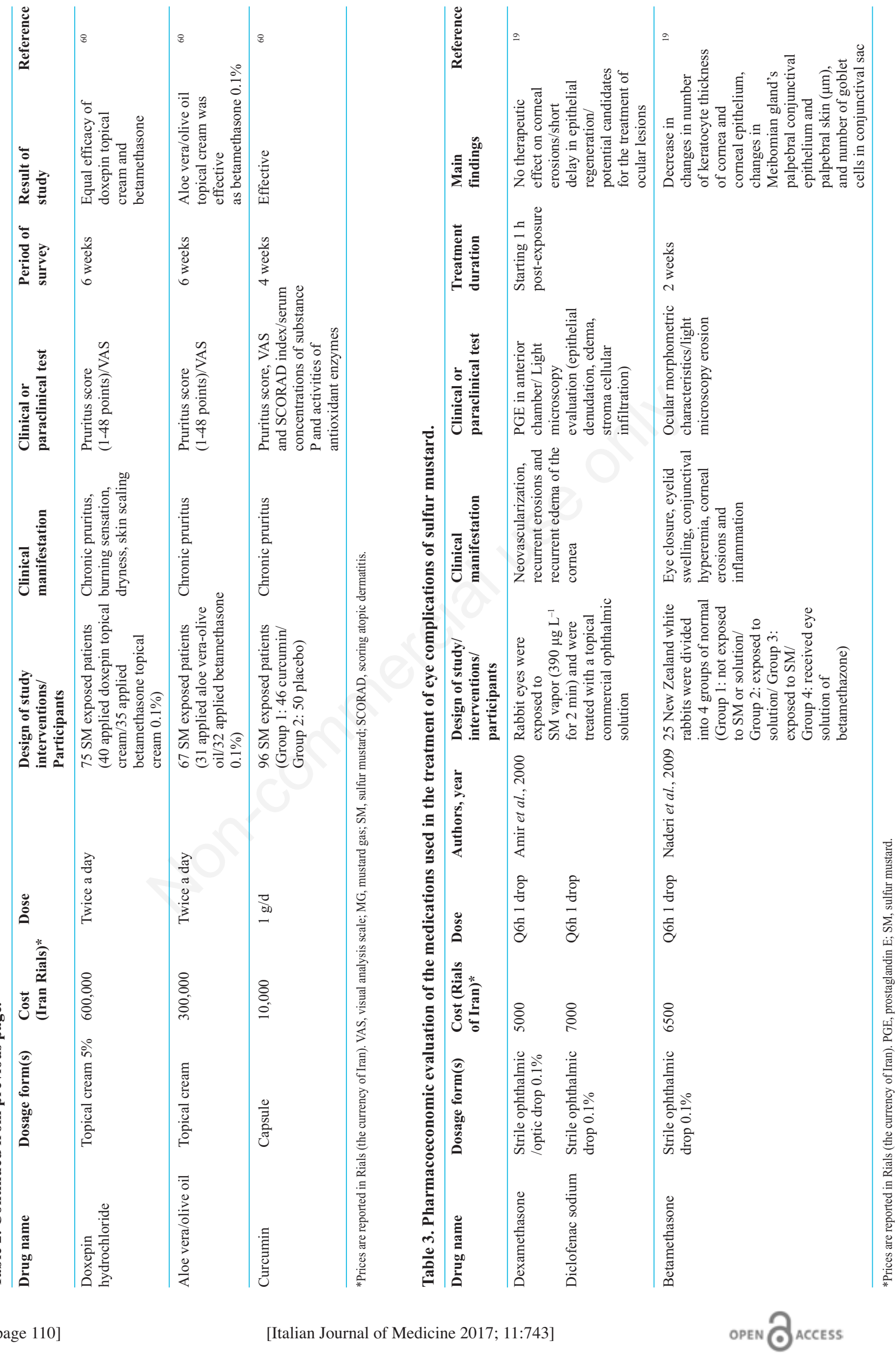


tients. As SM has a rapid and irreversible reaction with ocular tissue components, it may be useless to start washing after $10-15$ min post-exposure. ${ }^{2,19}$ Mild ocular injury could be treated with soothing eye solution used several times a day. Vaseline can prevent sticking of the eyelids together and is also useful to maintain drainage of ocular fluid. Topical antibiotics and mydriatics can prevent formation of synechiae. ${ }^{2,19,30}$ According to Kadar et al., only topical anti-inflammatory drugs have the criteria of an efficient post-exposure ocular treatment for SM injuries. ${ }^{69}$

In a study conducted by Gordon $\mathrm{et}$ al. on the effect of doxycycline on the injury of rabbit corneal organ cultures, results showed that eyes treated with doxycycline had better results than those received no therapy. Additionally, corneal thickness decreased somewhat faster using doxycycline drops, but the administration hydrogel formulation of doxycycline decreased the neovascularization. ${ }^{70}$ In another study, results showed that silibinin, a non-toxic natural flavanone, and a combination of doxycycline and dexamethasone are effective, more than doxycycline or dexamethasone alone, on SM analogue-induced ocular injuries by reversing epithelial thickening, microbullae formation and apoptotic cell death. Their results also showed strong multifunctional efficacy of silibinin in reversing SM-induced ocular injuries, which make it an effective and safe treatment for ocular injuries due to SM exposure. ${ }^{71}$ Another study on the effect of polyethylene glycol (PEG)-based doxycycline hydrogels on wound healing efficacy of doxycycline in SM analogues-exposed rabbit corneas in organ culture showed that doxycycline-PEG hydrogels accelerate corneal wound healing after vesicant injury. ${ }^{72}$

To date, there has been no definite treatment for SM-induced delayed keratopathy. However, artificial tears, therapeutic contact lenses, local/systemic corticosteroids and other immunosuppressive drugs such as azathioprin may be used according to keratitis severity. ${ }^{19}$

\section{Conclusions}

Reasonable prescription of drugs for respiratory, skin and ocular complications of SM needs awareness of the economic conditions of patients and cost-effectiveness of medications. Since patients have poor compliance with expensive drugs, it is not recommended to use costly and less effective medications for the treatment of SM complications. Unfortunately, many of the effective medications are not covered by the social insurance of armed forces especially for chemical injuries and these patients are not able to afford the necessary medications such as interferongamma and some of the effective topical creams. Health Policy should pay more attention to provide subsidized medications for chronic diseases like chronic complications of SM. For this reason, it is recommended that future studies evaluate the efficacies and costs of different brand name and generic drugs in relieving chronic complications of SM-exposed subjects. Finally, it is worth noting that the current available evidence is not strong enough to allow judgment on the efficacy of drugs in reducing the occurrence of hard outcomes in patients suffering from chronic respiratory complications of SM. Hence, future outcome trials are warranted to enable a better cost-effectiveness and pharmacoeconomic evaluation.

\section{References}

1. Ghabili K, Agutter PS, Ghanei M, et al. Mustard gas toxicity: the acute and chronic pathological effects. J Appl Toxicol 2010;30:627-43.

2. Balali-Mood M, Hefazi M. Comparison of early and late toxic effects of sulfur mustard in Iranian veterans. Basic Clin Pharmacol Toxicol 2006;99:273-82.

3. Papirmeister B, Feister AJ, Robinson SI, Ford RD. Medical defense against mustard gas: toxic mechanisms and pharmacological implications. Boca Raton, FL: CRC Press; 1991.

4. Dahl AR, Schlesinger RB, Heck HD, et al. Comparative dosimetry of inhaled materials: differences among animal species and extrapolation to man. Fundam Appl Toxicol 1991;16:1-13.

5. Afshinniaz F, Ghanei M. Relationship of the chronic respiratory symptoms with spirometric and laboratory parameters. Thesis Dissertation. Isfahan: Isfahan University of Medical Sciences; 1996.

6. Hankins J, Klotz W. Permanent pulmonary effects of gas in warfare. Am Rev Tuberc 1922;6:571.

7. Hosseini-khalili A, Haines DD, Modirian E, et al. Mustard gas exposure and carcinogenesis of lung. Mutat Res 2009;678:1-6.

8. Ghanei M, Khalili AR, Arab MJ, et al. Diagnostic and therapeutic value of short-term corticosteroid therapy in exacerbation of mustard gas-induced chronic bronchitis, Basic Clin Pharmacol Toxicol 2005;97:302-5.

9. Ghanei M, Panahi Y, Mojtahedzadeh M, et al. Effect of gamma interferon on lung function of mustard gas exposed patients, after 15 years. Pulm Pharmacol Ther 2006;19:148-53.

10. Ghanei M, Shohrati M, Harandi AA, et al. Inhaled corticosteroids and long-acting beta 2-agonists in treatment of patients with chronic bronchiolitis following exposure to sulfur mustard. Inhal Toxicol 2007;19:889-94.

11. Shohrati M, Aslani J, Eshraghi M, et al. Therapeutics effect of $\mathrm{N}$-acetyl cysteine on mustard gas exposed patients: evaluating clinical aspect in patients with impaired pulmonary function test. Respir Med 2008;102:443-8.

12. Ghanei M, Shohrati M, Jafari M, et al. N-acetylcysteine improves the clinical conditions of mustard gas-exposed patients with normal pulmonary function test. Basic Clin Pharmacol Toxicol 2008; 103:428-32.

13. McClintock SD, Hoesel LM, Das SK, et al. Attenuation 
of half sulfur mustard gas-induced acute lung injury in rats. J Appl Toxicol 2006;26:126-31.

14. Shohrati M, Ghanei M, Harandi AA, et al. Effect of nebulized morphine on dyspnea of mustard gas-exposed patients: a double-blind randomized clinical trial study. Pulm Med 2012;2012:610921.

15. Panahi Y, Motiei-Langroudi R, Alaeddini F, et al. Furosemide inhalation in dyspnea of mustard gas-exposed patients: a triple-blind randomized study. Inhal Toxicol 2008;20:873-7.

16. Boskabady MH, Attaran D, Shaffei MN. Airway responses to salbutamol after exposure to chemical warfare. Respirology 2008;13:288-93.

17. Willems J. Clinical management of mustard gas casualties. Ann Med Mil Belg 1989;3:1-61.

18. Kehe K, Thiermann H, Balszuweit F, et al. Acute effects of sulfur mustard injury--Munich experiences. Toxicology 2009;263:3-8.

19. Balali-Mood M, Hefazi M. The pharmacology, toxicology, and medical treatment of sulphur mustard poisoning. Fundam Clin Pharmacol 2005;19:297-315.

20. Shakarjian MP, Heck DE, Gray JP, et al. Mechanisms mediating the vesicant actions of sulfur mustard after cutaneous exposure. Toxicol Sci 2010;114:5-19.

21. Graham JS, Chilcott RP, Rice P, et al. Wound healing of cutaneous sulfur mustard injuries: strategies for the development of improved therapies. J Burns Wounds 2005;4:e1.

22. Mellor SG, Rice P, Cooper GJ. Vesicant burns. Br J Plast Surg 1991;44:434-7.

23. Helm U, Balali-Mood M. Cutaneous lesions produced by sulfur mustard. In: The First International Medical Congress on Chemical Warfare Agents in Iran. Mashhad: Mashhad University of Medical Sciences; 1988.

24. Naraghi ZS, Mansouri P, Mortazavi M. A clinicopathological study on acute cutaneous lesions induced by sulfur mustard gas (yperite). Eur J Dermatol 2005;15: 140-5.

25. Rowell M, Kehe K, Balszuweit F, et al. The chronic effects of sulfur mustard exposure. Toxicology 2009;263:9-11.

26. Shirazi S, Balali-Mood M. Comparison of early and late toxic effects of sulfur mustard poisoning in a two-year period. In: The First International Medical Congress on Chemical Warfare Agents in Iran. Mashhad: Mashhad University of Medical Sciences; 1988.

27. Balali-Mood M, Mousavi S, Balali-Mood B. Chronic health effects of sulphur mustard exposure with special reference to Iranian veterans. Emerg Health Threats J 2008;1:e7.

28. Saburi A, Shohrati M, Karbasi-Afshar R. Immune-based pathogenesis of sulfur mustard; much still need to be done! Iranian J Allergy Asthma Immunol 2012;11:349-50.

29. Mi L, Gong W, Nelson P, et al. Hypothermia reduces sulphur mustard toxicity. Toxicol Appl Pharmacol 2003;193:73-83.

30. Kehe K, Szinicz L. Medical aspects of sulphur mustard poisoning. Toxicology 2005;214:198-209.

31. Panahi Y, Davoodi SM, Khalili H, et al. Phenol and menthol in the treatment of chronic skin lesions following mustard gas exposure. Singapore Med J 2007; 48:392-5.

32. Sahebkar A, Cicero AF, Simental-Mendia LE, et al. Cur- cumin downregulates human tumor necrosis factoralpha levels: a systematic review and meta-analysis ofrandomized controlled trials. Pharmacol Research 2016 [Epub ahead of print].

33. Sahebkar A, Henrotin Y. Analgesic efficacy and safety of curcuminoids in clinical practice: a systematic review and meta-analysis of randomized controlled trials. Pain Med 2015 [Epub ahead of print].

34. Panahi Y, Hosseini MS, Khalili N, et al. Antioxidant and anti-inflammatory effects of curcuminoid-piperine combination in subjects with metabolic syndrome: a randomized controlled trial and an updated meta-analysis. Clin Nutr 2015;34:1101-8.

35. Panahi Y, Khalili N, Hosseini MS, et al. Lipid-modifying effects of adjunctive therapy with curcuminoidspiperine combination in patients with metabolic syndrome: results of a randomized controlled trial. Complement Ther Med 2014;22:851-7.

36. Panahi Y, Ghanei M, Bashiri S, et al. Short-term curcuminoid supplementation for chronic pulmonary complications due to sulfur mustard intoxication: positive results of a randomized double-blind placebo-controlled trial. Drug Res 2015;65:567-73.

37. Panahi Y, Badeli R, Karami GR, et al. Investigation of the efficacy of adjunctive therapy with bioavailabilityboosted curcuminoids in major depressive disorder. Phytother Res 2015;29:17-21.

38. Panahi Y, Rahimnia AR, Sharafi M, et al. Curcuminoid treatment for knee osteoarthritis: a randomized doubleblind placebo-controlled trial. Phytother Res 2014; 28:1625-31.

39. Sahebkar A. Are curcuminoids effective C-reactive protein-lowering agents in clinical practice? Evidence from a meta-analysis. Phytother Res 2014;28:633-42.

40. Sahebkar A, Mohammadi A, Atabati A, et al. Curcuminoids modulate pro-oxidant-antioxidant balance but not the immune response to heat shock protein 27 and oxidized LDL in obese individuals. Phytother Res 2013;27:1883-8.

41. Mohammadi A, Sahebkar A, Iranshahi M, et al. Effects of supplementation with curcuminoids on dyslipidemia in obese patients: a randomized crossover trial. Phytother Res 2013;27:374-9.

42. Sahebkar A. Molecular mechanisms for curcumin benefits against ischemic injury, Fertil Steril 2010;94:e7576; author reply e77.

43. Panahi Y, Saadat A, Beiraghdar F, et al. Adjuvant therapy with bioavailability-boosted curcuminoids suppresses systemic inflammation and improves quality of life in patients with solid tumors: a randomized doubleblind placebo-controlled trial. Phytother Res 2014; 28:1461-7.

44. Sahebkar A. Curcuminoids for the management of hypertriglyceridaemia. Nat Rev Cardiol 2014;11:123.

45. Mirzaei H, Naseri G, Rezaee R, et al. Curcumin: a new candidate for melanoma therapy? Int J Cancer 2016 [Epub ahead of print].

46. Momtazi AA, Derosa G, Maffioli P, et al. Role of microRNAs in the therapeutic effects of curcumin in noncancer diseases. Mol Diagn Ther 2016 [Epub ahead of print].

47. Panahi Y, Kianpour P, Mohtashami R, et al. Curcumin lowers serum lipids and uric acid in subjects with non- 
alcoholic fatty liver disease: a randomized controlled trial J Cardiovasc Pharmacol 2016 [Epub ahead of print].

48. Rahmani S, Asgary S, Askari G, et al. Treatment of nonalcoholic fatty liver disease with curcumin: a randomized placebo-controlled trial. Phytother Res 2016 [Epub ahead of print].

49. Sahebkar A, Chew GT, Watts GF. Recent advances in pharmacotherapy for hypertriglyceridemia. Progr Lipid Res 2014;56:47-66.

50. Panahi Y, Sahebkar A, Amiri M, et al. Improvement of sulphur mustard-induced chronic pruritus, quality of life and antioxidant status by curcumin: results of a randomised, double-blind, placebo-controlled trial. Br J Nutr 2012;108:1272-9.

51. Panahi Y, Sahebkar A, Parvin S, et al. A randomized controlled trial on the anti-inflammatory effects of curcumin in patients with chronic sulphur mustard-induced cutaneous complications. Ann Clin Biochem 2012; 49:580-8

52. Panahi Y, Ghanei M, Hajhashemi A, et al. Effects of curcuminoids-piperine combination on systemic oxidative stress, clinical symptoms and quality of life in subjects with chronic pulmonary complications due to sulfur mustard: a randomized controlled trial. J Diet Suppl 2016;13:93-105.

53. Shohrati M, Davoudi M, Almasi M, et al. Comparative study of Unna's Boot and betamethasone cream in the treatment of sulfur mustard-related pruritus. Cutan Ocul Toxicol 2007;26:303-9.

54. Shohrati M, Davoudi SM, Keshavarz S, et al. Cetirizine, doxepine, and hydroxyzine in the treatment of pruritus due to sulfur mustard: a randomized clinical trial. Cutan Ocul Toxicol 2007;26:249-55.

55. Shohrati M, Tajik A, Harandi AA, et al. Comparison of hydroxyzine and doxepin in treatment of pruritus due to sulfur mustard. Skinmed 2007;6:70-2.

56. Panahi Y, Moharamzad Y, Beiraghdar F, et al. Comparison of clinical efficacy of topical pimecrolimus with betamethasone in chronic skin lesions due to sulfur mustard exposure: a randomized, investigator-blind study. Basic Clin Pharmacol Toxicol 2009;104:171-5.

57. Panahi Y, Davoudi SM, Beiraghdar F, et al. Doxepin cream vs betamethasone cream for treatment of chronic skin lesions due to sulfur mustard. Skinmed 2011;9:152-8.

58. Panahi Y, Davoudi SM, Sahebkar A, et al. Efficacy of Aloe vera/olive oil cream versus betamethasone cream for chronic skin lesions following sulfur mustard exposure: a randomized double-blind clinical trial. Cutan Ocul Toxicol 2012;31:95-103.
59. Panahi Y, Sahebkar A, Davoudi SM, et al. Efficacy and safety of immunotherapy with interferon-gamma in the management of chronic sulfur mustard-induced cutaneous complications: comparison with topical betamethasone 1\%. Sci World J 2012;2012:285274.

60. Panahi Y, Davoudi SM, Moharamzad Y, et al. Comparison of topical capsaicin and betamethasone in the treatment of chronic skin lesions due to sulfur mustard exposure. Cutan Ocul Toxicol 2008;27:203-11.

61. Etezad-Razavi M, Mahmoudi M, Hefazi M, et al. Delayed ocular complications of mustard gas poisoning and the relationship with respiratory and cutaneous complications. Clin Exper Ophthalmol 2006;34:342-6.

62. Solberg Y, Alcalay M, Belkin M. Ocular injury by mustard gas. Surv Ophthalmol 1997;41:461-6.

63. Javadi MA, Yazdani S, Sajjadi H, et al. Chronic and delayed-onset mustard gas keratitis: report of 48 patients and review of literature. Ophthalmology 2005; 112:617-25.

64. Vidan A, Luria S, Eisenkraft A, et al. Ocular injuries following sulfur mustard exposure: clinical characteristics and treatment. Isr Med Assoc J 2002;4:577-8.

65. Ghassemi-Broumand M, Aslani J, Emadi SN. Delayed ocular, pulmonary, and cutaneous complications of mustards in patients in the city of Sardasht, Iran. Cutan Ocul Toxicol 2008;27:295-305.

66. Safaei A, Saluti R, Kumar PV. Conjunctival dysplasia in soldiers exposed to mustard gas during the Iraq-Iran war: scrape cytology. Acta Cytol 2001;45:909-13.

67. Pleyer U, Sherif Z, Baatz H, et al. Delayed mustard gas keratopathy: clinical findings and confocal microscopy. Am J Ophthalmol 1999;128:506-7.

68. Aasted A, Darre E, Wulf HC. Mustard gas: clinical, toxicological, and mutagenic aspects based on modern experience. Ann Plast Surg 1987;19:330-3.

69. Kadar T, Dachir S, Cohen L, et al. Ocular injuries following sulfur mustard exposure--pathological mechanism and potential therapy. Toxicology 2009;263:59-69.

70. Gordon MK, Desantis A, Deshmukh M, et al. Doxycycline hydrogels as a potential therapy for ocular vesicant injury. J Ocul Pharmacol Ther 2010;26:407-19.

71. Tewari-Singh N, Jain AK, Inturi S, et al. Silibinin, dexamethasone, and doxycycline as potential therapeutic agents for treating vesicant-inflicted ocular injuries. Toxicol Appl Pharmacol 2012;264:23-31.

72. Anumolu SS, DeSantis AS, Menjoge AR, et al. Doxycycline loaded poly(ethylene glycol) hydrogels for healing vesicant-induced ocular wounds. Biomaterials 2010;31:964-74. 